\title{
Malignant External Otitis with Facial Nerve Paralysis
}

\author{
Giancarlo Tirelli, Annalisa Gatto, Silvia Brancatelli, Alice Piccinato \\ ENT Clinic, Head and Neck Department, Azienda Sanitaria Universitaria Integrata di Trieste, Trieste, Italy
}

\section{Abstract}

Malignant external otitis (MOE) is a severe infection of the external auditory canal and the skull base that is often seen in elderly diabetic patients with a high mortality and morbidity rate. in $>98 \%$ of cases, the causative pathogen is Pseudomonas aeruginosa. Here, we describe a case of MOE associated with facial nerve paralysis in a patient hospitalized. A 62-year-old man was referred to our department experiencing right otalgia, purulent otorrhea, ear loss, and a Grade III right facial nerve palsy according to the House-Brackmann scale. He was affected by uncompensated insulin-dependent type 2 diabetes. The patient had a history of noncholesteatomatous ipsilateral chronic otitis treated with tympanomastoid surgery 10 years before, without any sign of recurrence. After 10 days of specific antibiotic and corticosteroid therapy, symptoms were reduced and the paralysis of the superior branches of the facial nerve had improved, but the persisting swell of the ear canal did not allow a thorough evaluation of the tympanic membrane. Therefore, an explorative tympanotomy was performed, and few days after surgery, the marginalis branch paralysis of the facial nerve had improved according toelectroneurography (ENG) results, and the culture swab showed a polymicrobial flora.

Keywords: External auditory canal, facial nerve paralysis, malignant external otitis, otitis, tympanotomy

\section{INTRODUCTION}

Malignant external otitis (MOE) is a severe infection of the external auditory canal and the skull base that is often seen in elderly diabetic patients with a high mortality and morbidity rate: in more than $98 \%$ of cases the causative pathogen is $P$. Aeruginosa.

Usually the disease begins as an infection of the soft tissues of the external auditory canal and then spreads to the temporal bone, involving stylomastoid and jugular foramina and eventually affecting cranial nerve function.

Its treatment requires prolonged intravenous antibiotic therapy, regular aural toilet, strict glycaemic control and in some cases a surgical courettage. ${ }^{[1]}$

We report a case of a patient who presented MOE associated with facial nerve paralysis.

\section{Case Report}

A 62-year-old man was referred to our department experiencing right otalgia, purulent otorrhea, ear loss, and a Grade III right facial nerve palsy according to the HouseBrackmann scale.

\begin{tabular}{|c|c|}
\hline Submission: 26-February-2019 & pted: 13-July-2019 Published: 04-December-2C \\
\hline & ss this article online \\
\hline 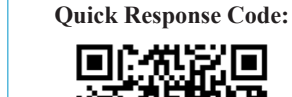 & $\begin{array}{l}\text { Website: } \\
\text { www.indianjotol.org }\end{array}$ \\
\hline 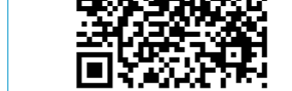 & $\begin{array}{l}\text { DOI: } \\
\text { 10.4103/indianjotol.INDIANJOTOL_25_19 }\end{array}$ \\
\hline
\end{tabular}

He was affected by decompensated insulin-dependent type 2 diabetes. The patient had a history of noncholesteatomatous ipsilateral chronic otitis treated with tympanomastoid surgery 10 years before, without any sign of recurrence.

At the examination, there was a purulent discharge from the right ear, the canal appeared red and swollen, the tympanic membrane was not visible, and the retroauricular and preauricular areas showed redness and swelling. Meningeal signs were negative, without any cervical lymphadenopathy or any alteration of the blood examinations.

Computed tomography (CT) scan showed opacification of the right middle ear and mastoid cavity, with osteolysis of the facial canal and of the tegmen tympani, whereas magnetic resonance imaging (MRI) with contrast showed a mild enhancement in the meninx.

The scintigraphic evaluation with Technetium-99 (Tc-99m) confirmed osteomyelitis with an increased uptake of radio-marked difosfonate in the temporomandibular joint,

Address for correspondence: Prof. Silvia Brancatelli, ENT Clinic, Head and Neck Department, Azienda Sanitaria Universitaria Integrata di Trieste, Trieste, Italy. E-mail: silvia.brancatelli@hotmail.it

This is an open access journal, and articles are distributed under the terms of the Creative Commons Attribution-NonCommercial-ShareAlike 4.0 License, which allows others to remix, tweak, and build upon the work non-commercially, as long as appropriate credit is given and the new creations are licensed under the identical terms.

For reprints contact: reprints@medknow.com

How to cite this article: Tirelli G, Gatto A, Brancatelli S, Piccinato A. Malignant external otitis with facial nerve paralysis. Indian J Otol 2019;25:216-8. 
mastoid cells, semicircular canals, cochlea, middle and inner ear, and in the greater wing of the sphenoid bone.

The first antibiogram was positive for Pseudomonas aeruginosa, and according to the infectivologist's evaluation, a specific therapy with intravenous $400 \mathrm{mg}$ ciprofloxacin every $12 \mathrm{~h}$ was undertaken. We also started a corticosteroid therapy with the support of a diabetes specialist.

Due to the MRI results, we asked for a neurological evaluation; a rachicentesis was performed excluding the hypothesis of meningitis. The Electromyography (emg) and ENG confirmed the right seventh nerve palsy with a decreased conduction velocity of all the branches of the nerve.

Hyperbaric oxygen therapy was not undertaken because of the lack of a clear evidence demonstrating its efficacy (level of evidence Grade D). ${ }^{[1]}$ We took a biopsy of a polypoid formation of the ear canal that resulted to be hyperplastic tissue with dyskeratosis and granulation tissue.

After 10 days of therapy, symptoms were reduced and the paralysis of the superior branches of the facial nerve had mildly improved, but the persisting swell of the ear canal did not allow a thorough evaluation of the tympanic membrane. Therefore, an explorative tympanotomy was performed, without any sign of otorrhea, osteolysis, or cholesteatoma, but it was determined. Few days after surgery, the marginalis branch paralysis of the facial nerve had improved according to ENG results, and the culture swab showed a polymicrobial flora. Moreover, the swelling of the auditory canal was reduced.

The antibiotic therapy was carried on after the dismission with an association of oral amoxicillin-clavulanic acid $1 \mathrm{~g} 3 /$ die and ciprofloxacin $500 \mathrm{mg}$ 2/die for 4 weeks. The weekly follow-up showed an improvement of the edema, and after 1 month, the symptomatology disappeared and the facial nerve function had further ameliorated according to ENG and clinical signs.

After 3 months, at the scintigraphic control with marked Tc$99 \mathrm{~m}$. leukocytes, the inflammation and the facial nerve palsy were solved.

The patient consent form for this paper was obtained.

\section{Discussion}

Toulmouche reported the first case of malignant external otitis (MOE) in 1838. In 1959, MELTZER reported a case of pseudomonal osteomyelitis of the temporal bone, but the name was coined by Chandler who defined MOE as an unresponsive infection of the external ear canal associated with granulation tissue at the junction of the osseous and cartilaginous portions that occurs almost exclusively in elderly and diabetic patients.

Most cases of MOE ( $86 \%-90 \%)$ have been reported in diabetic patients. This is probably due to immunodeficiency and to abnormalities of small blood vessels.

There is also a group of nondiabetic immunocompromised patients who can develop the disease (e.g., malignancy, chemotherapy, and malnutrition). Even if the most common causative organism of MOE is $P$. aeruginosa, there are other organisms that have been isolated, such as Proteus mirabilis, Aspergillus fumigatus, Proteus spp., Klebsiella spp., and staphylococci. The majority of fungal MOE occurs in immunosuppressed individuals with AIDS. ${ }^{[2]}$

The symptoms of MOE are severe unremitting throbbing otalgia, purulent otorrhea, the sensation of a blocked ear, and hearing loss.

Cranial nerves can be affected by inflammation, and the facial nerve (VII) is the most commonly affected.

In 1987, Cohen and Friedman listed a set of major and minor criteria for MOE and suggested that the diagnosis was established when all the major criteria were present.

The major criteria were as follows: pain, edema, exudate, granulations, microabscess (when operated), positive bone scan or failure of local treatment often $>1$ week, and possibly Pseudomonas spp. in culture. The minor criteria were as follows: diabetes, cranial nerve involvement, positive radiograph, debilitating condition, and old age. All of the obligatory criteria must be present in order to establish the diagnosis.

Levenson's criteria can also be used for diagnosis. Criteria include refractory otitis externa, severe nocturnal otalgia, and purulent otorrhea associated with Pseudomonas infection and granulation tissue in an immunocompromised or diabetic patient. $^{[3]}$

Both Levenson's and Cohen and Friedman's criteria were present in our patient.

Laboratory parameters are generally normal in MOE, with the exception of an elevated erythrocyte sedimentation rate and/or C-reactive protein.

The leukocyte count in MOE is usually normal or mildly elevated.

CT scanning is ideal for the assessment of bone erosion in MOE, whereas MRI results are better than CT at demonstrating medial skull base disease due to its ability to delineate changes in the fat content of the marrow. ${ }^{[4]}$

Gallium citrate scanning can be used to follow disease activity, since the radioisotope is incorporated into granulocytes and bacteria.

Bone scanning with technetium (Tc-99m), where the radionuclide tracer accumulates at sites of osteoblastic activity, is a very sensitive examination, but it is not specific, since there are reports of positive bone scans in simple external otitis, and bone scans are not suitable for the following response to treatment since they do not normalize.

A biopsy is indicated in order to differentiate MOE from squamous cell carcinoma of the external auditory canal.

The treatment of MOE mainly consists of strict glycemic control, aural toilet, systemic and ototopic antimicrobial therapy, and hyperbaric oxygen therapy. ${ }^{[2]}$ 
The use of fluoroquinolones (especially ciprofloxacin monotherapy) or ceftazidime monotherapy has been proved to be very effective.

Patients with ciprofloxacin-resistant $P$. aeruginosa require parenteral antibiotics with antipseudomonal beta-lactam antibiotics with or without an aminoglycoside. ${ }^{[5]}$

The antibiotic treatment should be carried on until the resolution of osteomyelitis. The response to the treatment can be evaluated with a gallium citrate Ga 67 scan, which should be repeated every 4-6 weeks, until the normalization of the examination.

Hyperbaric oxygen therapy should be used only as an adjunct to antimicrobial therapy, especially for those patients experiencing a poor response to therapy or with recurrent cases. ${ }^{[3,6]}$

Surgery is indicated for local debridement, removal of necrotic bone tissue, or abscess drainage, whereas facial nerve surgical decompression is not indicated.

MOE is characterized by a recurrence of $9 \%-27 \%$, and this is usually related to the inadequate length of the therapy.

Its complications include meningitis, abscess, and sagittal, dural, and cavernous sinus thrombosis.

Most current studies report a mortality rate of $<10 \%$, but it remains high in patients with cranial neuropathies (other than VII), intracranial complications, or irreversible systemic immunosuppression.

\section{CONCLUSION}

Malignant otitis externa is an aggressive infection that can represent a life-threatening condition.

In our patient, a prolonged steroid and antibiotic treatment and a strict control of glycemic levels permitted to control osteomyelitis, but the surgical approach appeared essential for the resolution of the disease.
The aural toilet with the elimination of the necrotic and granulation tissues leads in fact to the aeration of the tympanic cavity, helping the resolution of the paralysis, especially the function of the marginal branch.

In our patient, we obtained the complete resolution of the infection without any recurrence over an year confirming, according to the literature, that the presence of the facial paralysis does not represent a negative prognostic factor.

\section{Declaration of patient consent}

The authors certify that they have obtained all appropriate patient consent forms. In the form the patient(s) has/have given his/her/their consent for his/her/their images and other clinical information to be reported in the journal. The patients understand that their names and initials will not be published and due efforts will be made to conceal their identity, but anonymity cannot be guaranteed.

\section{Financial support and sponsorship}

Nil.

\section{Conflicts of interest}

There are no conflicts of interest.

\section{ReFERENCES}

1. Narozny W, Kuczkowski J, Stankiewicz C, Kot J, Mikaszewski B, Przewozny T. Value of hyperbaric oxygen in bacterial and fungal malignant external otitis treatment. Eur Arch Otorhinolaryngol 2006;263:680-4.

2. Rubin Grandis J, Branstetter BF $4^{\text {th }}$, Yu VL. The changing face of malignant (necrotising) external otitis: Clinical, radiological, and anatomic correlations. Lancet Infect Dis 2004;4:34-9.

3. Rutka J. Acute otitis externa: Treatment perspectives. Ear Nose Throat J 2004;83:20-1.

4. Ismail H, Hellier WP, Batty V. Use of magnetic resonance imaging as the primary imaging modality in the diagnosis and follow-up of malignant external otitis. J Laryngol Otol 2004;118:576-9.

5. Berenholz L, Katzenell U, Harell M. Evolving resistant pseudomonas to ciprofloxacin in malignant otitis externa. Laryngoscope 2002;112:1619-22.

6. Ling SS, Sader C. Fungal malignant otitis externa treated with hyperbaric oxygen. Int J Infect Dis 2008;12:550-2. 